\title{
Mod 2 normal numbers and skew products
}

\author{
by \\ Geon Ho Choe (Daejeon), Toshihiro Hamachi (Fukuoka) and \\ Hitoshi NAKADA (Yokohama)
}

\begin{abstract}
Let $E$ be an interval in the unit interval [0,1). For each $x \in[0,1)$ define $d_{n}(x) \in\{0,1\}$ by $d_{n}(x):=\sum_{i=1}^{n} 1_{E}\left(\left\{2^{i-1} x\right\}\right)(\bmod 2)$, where $\{t\}$ is the fractional part of $t$. Then $x$ is called a normal number mod 2 with respect to $E$ if $N^{-1} \sum_{n=1}^{N} d_{n}(x)$ converges to $1 / 2$. It is shown that for any interval $E \neq(1 / 6,5 / 6)$ a.e. $x$ is a normal number mod 2 with respect to $E$. For $E=(1 / 6,5 / 6)$ it is proved that $N^{-1} \sum_{n=1}^{N} d_{n}(x)$ converges a.e. and the limit equals $1 / 3$ or $2 / 3$ depending on $x$.
\end{abstract}

1. Introduction. Let $(X, \mu)$ be a probability space and let $T: X \rightarrow X$ be an ergodic transformation. Given a measurable subset $E \subset X$, we consider the binary sequence $d_{n}(x) \in\{0,1\}$ defined by

$$
d_{n}(x):=\sum_{i=1}^{n} 1_{E}\left(T^{i-1} x\right)(\bmod 2)
$$

where $1_{E}$ is the characteristic function of $E$. The mod 2 normality problem is to investigate the convergence of

$$
\lim _{N \rightarrow \infty} \frac{1}{N} \sum_{n=1}^{N} d_{n}(x)
$$

to the limit $1 / 2$ or the convergence of

$$
\lim _{N \rightarrow \infty} \frac{1}{N} \sum_{n=1}^{N} e_{n}(x)
$$

to the limit 0 , where $e_{n}(x)=\exp \left(\pi i d_{n}(x)\right)$.

2000 Mathematics Subject Classification: Primary 28D05, 37A30, 11K16.

Key words and phrases: ergodicity, mod 2 normal number, skew product, coboundary.

The initial stage of work was done while the authors were visiting the Erwin Schrödinger Institute.

Supported by Korea Research Foundation Grant (KRF-2003-041-C00020).

Editorial note: The first version of this paper was received by the Editors on September 30, 1999 under a different title. 
When $T$ is given by an irrational translation on the unit interval $[0,1)$, i.e., $T x=\{x+\theta\}, \theta$ irrational, the problem was first investigated by Veech [14] using Furstenberg's idea on coboundaries (see [6]). Let $E$ be an interval. Define

$$
\mu_{\theta}(E)=\lim _{N \rightarrow \infty} \frac{1}{N} \sum_{n=1}^{N} d_{n}(x)
$$

if the limit exists. Let $t$ be the length of $E$. It can be shown that for arbitrary $\theta$ if $t \in 2 \mathbb{Z} \cdot \theta+\mathbb{Z}$ then $\mu_{\theta}(E)$ exists but it may not be equal to $1 / 2$. And for arbitrary $\theta$ and $t$ at least one of $\mu_{\theta}(E)$ or $\mu_{\theta}([0,1] \backslash E)$ is $1 / 2$, the limit existing. Veech proved that $\mu_{\theta}(E)$ exists for every interval $E$ if and only if $\theta$ has bounded partial quotients in its continued fraction expansion, and in this case if $t \notin 2 \mathbb{Z} \cdot \theta+\mathbb{Z}$ then $\mu_{\theta}(E)=1 / 2$. For closely related results, see [8], [9], [11], [13], [15].

In this article we are interested in the case that $X=[0,1)$ and $T x=$ $\{2 x\}$, where $\{t\}$ is the fractional part of $t$. Set inclusions and identities are understood modulo measure zero sets.

Definition 1.1. Let $E$ be a measurable subset of $[0,1)$. For $x \in[0,1)$ define $d_{n}(x) \in\{0,1\}$ as above. Then $x$ is called a normal number mod 2 with respect to $E$ if $N^{-1} \sum_{n=1}^{N} d_{n}(x)$ converges to $1 / 2$.

We state the main result that will be proved in Section 3 .

Main Theorem. (i) For any interval $E \neq(1 / 6,5 / 6)$ a.e. $x \in[0,1)$ is a normal number mod 2 with respect to $E$.

(ii) For $E=(1 / 6,5 / 6)$ a.e. $x \in[0,1)$ is not a normal number mod 2 with respect to $E$. More precisely, for $E=(1 / 6,5 / 6)$ we have

$$
\begin{array}{ll}
\lim _{N \rightarrow \infty} \frac{1}{N} \sum_{n=1}^{N} d_{n}=\frac{2}{3} & \text { for a.e. } x \in\left[\frac{1}{3}, \frac{2}{3}\right] \\
\lim _{N \rightarrow \infty} \frac{1}{N} \sum_{n=1}^{N} d_{n}=\frac{1}{3} & \text { for a.e. } x \in\left[0, \frac{1}{3}\right] \cup\left[\frac{2}{3}, 1\right] .
\end{array}
$$

2. $\mathbb{Z}_{2}$-extensions. Let $T$ be ergodic on a probability space $(X, \mu)$. It is not necessarily invertible. Let $G$ be a compact abelian group. Suppose that $\phi: X \rightarrow G$ is measurable. Define a skew product $T_{\phi}$ on $X \times G$ by $T_{\phi}(x, y)=(T x, \phi(x)+y)$. It is also called a $G$-extension of $T$ by $\phi$.

Definition 2.1. A function $\phi: X \rightarrow G$ is called a $G$-coboundary if there exists a measurable function $q: X \rightarrow G$ satisfying $\phi(x)=q(x)-q(T x)$.

From now on we consider the case $G=\mathbb{Z}_{2}=\{0,1\}$. Put $\phi=1_{E}$. Define $V_{\phi}$ on $L^{2}(X)$ by $\left(V_{\phi} f\right)(x)=e^{\pi i \phi(x)} f(T x)$. Since $\left(V_{\phi}\right)^{n} 1=e_{n}$, von 
Neumann's Mean Ergodic Theorem implies that $N^{-1} \sum_{n=1}^{N} e_{n}$ converges to the orthogonal projection of 1 onto the subspace $H=\left\{h: V_{\phi} h=h\right\}$. Now for the pointwise convergence the following is known. For the proof see [3].

FACT 2.2. (i) Suppose that $T_{\phi}: X \times \mathbb{Z}_{2} \rightarrow X \times \mathbb{Z}_{2}$ is ergodic. Then for a.e. $x$,

$$
\lim _{N \rightarrow \infty} \frac{1}{N} \sum_{n=1}^{N} e_{n}(x)=0 .
$$

(ii) Suppose that $T_{\phi}$ is not ergodic. Then for a.e. $x$,

$$
\lim _{N \rightarrow \infty} \frac{1}{N} \sum_{n=1}^{N} e_{n}(x)=h(x) \int_{X} h d \mu,
$$

where $e^{\pi i \phi(x)}=h(x) h(T x)$ and $h(x) \in\{ \pm 1\}$ for a.e. $x$.

If $\phi$ is a coboundary then it is possible that $H$ is not trivial and the limit is not zero (see [5]). In this paper we investigate for which $E$ the skew product is ergodic. The following fact is well known.

FACT 2.3. Let $T$ be ergodic on $X$. Suppose $\phi(x) \in \mathbb{Z}_{2}$, or equivalently $\phi=1_{E}$ for some measurable subset $E$. Then the following are equivalent:

(i) $T_{\phi}$ is not ergodic on $X \times \mathbb{Z}_{2}$.

(ii) There exists a $\mathbb{Z}_{2}$-valued measurable function $q$ such that $\phi(x)=$ $q(x)+q(T x)(\bmod 2)$. In this case, $q=1_{F}$ for some measurable $F$.

(iii) $E=F \triangle T^{-1} F$ for some measurable $F$, where $\triangle$ denotes the symmetric difference.

The following fact is a special case of a well known result (see Zimmer [16] and also [10], [7]). We give a proof for the sake of completeness. Throughout the rest of the article addition is done modulo 2 .

FACT 2.4. Let $T$ be ergodic on $X$ and let $\phi$ be $\mathbb{Z}_{2}$-valued on $X$. If $T_{\phi}$ is not ergodic, then it has exactly two ergodic components, each having the same measure. In this case the two ergodic components of $T_{\phi}$ are $\{(x, q(x))$ : $x \in X\}$ and $\{(x, 1+q(x)): x \in X\}$, where $q$ is $\mathbb{Z}_{2}$-valued and $\phi(x)=$ $q(x)+q(T x)$.

Proof. Since $T_{\phi}$ is not ergodic, Fact 2.3 implies that $\phi$ is a coboundary. Define $Q$ and $S$ on $X \times \mathbb{Z}_{2}$ by $Q(x, y)=(x, q(x)+y)$ and $S(x, y)=(T x, y)$. Then $Q^{-1}=Q$ and $Q \circ T_{\phi}=S \circ Q$. Note that $T_{\phi}$ is isomorphic to $S$, which has two ergodic components $X \times\{0\}$ and $X \times\{1\}$. Therefore the ergodic components of $T_{\phi}$ are $Q(X \times\{0\})=\{(x, q(x)): x \in X\}$ and $Q(X \times\{1\})=$ $\{(x, 1+q(x)): x \in X\}$. 
3. Construction of piecewise linear maps. In this section we study $\mathbb{Z}_{2}$-extensions of $T: x \mapsto\{2 x\}$ on $X=[0,1)$ from the viewpoint of interval mappings. The subsets $X_{0}=X \times\{0\}$ and $X_{1}=X \times\{1\}$ are identified with the intervals $[0,1)$ and $[1,2)$, respectively, and $T_{\phi}$ is regarded as being defined on $\widetilde{X}=[0,2)$. The new interval map on $\widetilde{X}$ induced from $T_{\phi}$ is denoted by $\widetilde{T}_{\phi}$. If $\widetilde{T}_{\phi}$ is not ergodic, then it has two ergodic components in $\widetilde{X}$, each having Lebesgue measure 1 .

More precisely, for a measurable subset $E \subset[0,1)$, put $\phi=1_{E}$. Recall that $T_{\phi}(x, i)=(T x, i+\phi(x)), i \in\{0,1\}$. Define $\psi: X \times\{0,1\} \rightarrow[0,2)$ by $\psi(x, i)=x+i$. For $u \in[0,2)$ let

$$
\widetilde{T}_{\phi}(u)=\psi \circ T_{\phi} \circ \psi^{-1}(u) .
$$

Then $\widetilde{T}_{\phi}$ preserves Lebesgue measure and satisfies

$$
\widetilde{T}_{\phi}(u)= \begin{cases}\{2 u\}+1_{E}(u), & 0 \leq u<1, \\ \{2 u\}+1-1_{E}(u-1), & 1 \leq u<2 .\end{cases}
$$

EXAMPle 3.1 (An ergodic skew product). Take $E=[1 / 2,1]$ and $\phi=1_{E}$. Then $\widetilde{T}_{\phi}$ can be regarded as the piecewise linear map given in Fig. 1. Note that it is ergodic. See the Folklore Theorem given in [1].

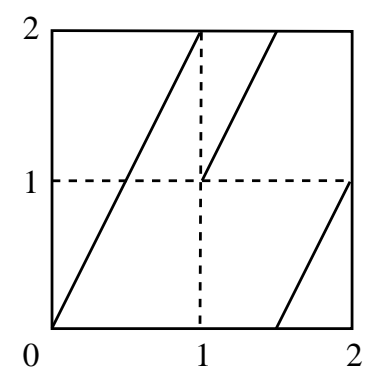

Fig. 1. An ergodic skew product $T_{\phi}$ generated by the interval $E=[1 / 2,1) \subset X=[0,1)$ is regarded as an interval map $\widetilde{T}_{\phi}$ on $\widetilde{X}=[0,2)$.

EXAmple 3.2 (A nonergodic skew product). Take $E=[1 / 4,3 / 4]$ and $\phi=1_{E}$. Put $F=[1 / 2,1]$ and $q=1_{F}$. Then $E=F \triangle T^{-1} F$ and $\phi$ is a coboundary. Then $\widetilde{T}_{\phi}$ can be regarded as the piecewise linear map given in Fig. 2. It has two ergodic components: $[0,1 / 2) \cup[3 / 2,2)$ and $[1 / 2,3 / 2)$. This can be seen from Fact 2.4.

Let $J$ be an interval and $\tau$ be a piecewise $C^{2}$ mapping on $J$. Assume that $\inf _{x \in J_{1}}\left|\tau^{\prime}(x)\right|>1$, where $J_{1}=\left\{x \in J: \tau^{\prime}(x)\right.$ exists $\}$. The points of $J-J_{1}$ are called the points of discontinuity. For $x \in J$, let $\Lambda(x)$ be the set of limit points of $\tau^{n}(x)$, that is, $\Lambda(x)=\bigcap_{N=1}^{\infty} \overline{\left\{\tau^{n}(x): n \geq N\right\}}$. Note that $\tau(\Lambda(x))=\Lambda(x)$. Li and Yorke [12] proved the following. 


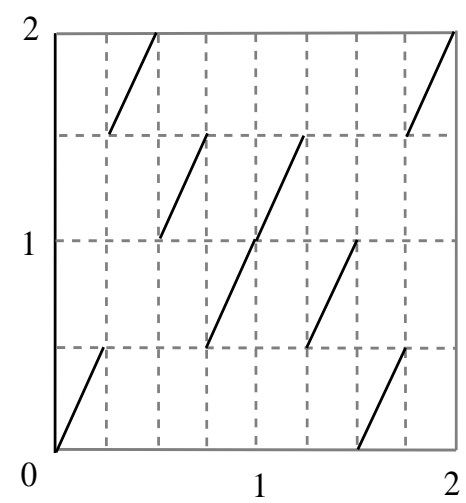

Fig. 2. A nonergodic skew product $T_{\phi}$ generated by the interval $E=[1 / 4,3 / 4) \subset X=$ $[0,1)$ is regarded as an interval map $\widetilde{T}_{\phi}$ on $\widetilde{X}=[0,2)$. It has two ergodic components: $K_{1}=[1 / 2,3 / 2]$ and $K_{2}=\tilde{X}-K_{1}$.

FACT 3.3. For a.e. $x, \Lambda(x)$ is the union of (one or more) intervals of positive length. Furthermore, there is a finite collection of sets $L_{1}, \ldots, L_{n}$, where each $L_{i}$ is a union of finitely many disjoint intervals, such that for a.e. $x, \Lambda(x)$ is one of the sets $L_{i}$. (The sets $L_{i}$ are ergodic components of $\tau$.) If $i \neq j$ then $L_{i} \cap L_{j}$ contains at most a finite number of points and each $L_{i}$ contains in its interior a point of discontinuity of $\tau$ and/or $\tau^{\prime}$. For each $L_{i}$ there exists a unique absolutely continuous $\tau$-invariant measure $\mu_{i}$ such that $\mu_{i}\left(L_{i}\right)=1$ and $\mu_{i}\left(L_{j}\right)=0$ for $j \neq i$.

REMarK 3.4. Suppose that $E$ is an interval. Then we have the following: (i) $\widetilde{T}_{\phi}$ is piecewise linear and satisfies $\widetilde{T}_{\phi}^{\prime}(x)=2$ except at finitely many points. (ii) If $\widetilde{T}_{\phi}$ is not ergodic, then an ergodic component is a union of finitely many disjoint intervals. More precisely, if $E=F \triangle T^{-1} F$, then Fact 2.4 implies that the ergodic components are given by $([0,1) \backslash F) \cup(F+1)$ and $F \cup([1,2) \backslash(F+1))$.

Proposition 3.5. Suppose $E$ is a finite union of nonempty open intervals such that $E \subset[0,1 / 2)$ or $E \subset[1 / 2,1)$. Then $T_{\phi}$ is ergodic.

Proof. First, consider the case $E \subset[1 / 2,1)$. We regard $T_{\phi}$ as a map $\widetilde{T}_{\phi}$ on $[0,2)$. Note that an ergodic component has Lebesgue measure 1 or 2 . Since $E$ is a union of finitely many intervals, $\widetilde{T}_{\phi}$ is an interval map on $[0,2)$ that satisfies the condition from Fact 3.3. This implies that an ergodic component of $\widetilde{T}_{\phi}$ is a union of finitely many intervals. Thus $\left[0,1 / 2^{n}\right)$ is in an ergodic component of $\widetilde{T}_{\phi}$ for sufficiently large $n$. This implies that this ergodic component contains $(0,1)$. Note that the intersection of $\widetilde{T}_{\phi}([0,1))$ and $[1,2)$ is of positive measure. Consequently, the Lebesgue measure of the 
ergodic component is greater than 1, which implies that the measure is equal to 2 . Thus there is only one ergodic component.

Next, consider $E \subset[0,1 / 2)$. In this case we start with $\left(1-1 / 2^{n}, 1\right)$, and proceed as before.

The following example shows that the above theorem cannot be extended to general measurable sets.

ExAmple 3.6. Put $I=[3 / 4,1)$ and $F=\bigcup_{k=0}^{\infty} 2^{-k} I$. Let $q=1_{F}$. Then $q(x)+q(T x)=1_{E}(x)(\bmod 2)$ for $E=F \triangle T^{-1} F \subset[1 / 2,7 / 8]$. In this case $T_{\phi}$ is not ergodic.

Using $\widetilde{T}_{\phi}$ we obtain the following result. A similar result was also obtained by Ahn [2]. His proof uses the argument previously employed in [3], [4], where the problem was investigated for intervals with dyadic rational endpoints.

Proposition 3.7. Let $E$ be an interval in $[0,1)$ of length less than 1 , and let $\phi=1_{E}$. Then $T_{\phi}$ is not ergodic if and only if $E=(1 / 4,3 / 4)$ or $E=(1 / 6,5 / 6)$.

Proof. If $E=(1 / 4,3 / 4)$ or $E=(1 / 6,5 / 6)$, then $E=F \triangle T^{-1} F$ for $F=(1 / 3,2 / 3)$ or $F=(1 / 2,1)$, respectively. So $T_{\phi}$ is not ergodic by Fact 2.3. Conversely, assume that $T_{\phi}$ is not ergodic. Choose $F$ such that $E=F \triangle T^{-1} F$. Note that $F$ is a disjoint union of $n$ intervals for some $n \geq 1$ by Fact 3.3. Here a set of the form $(\alpha, \beta) \cup(\beta, \gamma)$ is regarded as a single interval $(\alpha, \gamma)$. We may assume that $F \subset(\delta, 1)$ for some $\delta>0$ by taking its complement if necessary. Note that

$$
T^{-1} F \subset(\delta / 2,1 / 2) \cup(1 / 2+\delta / 2,1) .
$$

Since $F$ has $2 n$ boundary points, $T^{-1} F$ has $4 n$ boundary points, hence the interval $F \triangle T^{-1} F$ has at least $2 n$ boundary points. This is possible only if $n=1$, so $F$ is an interval of the form $F=(a, b), a>0$. Then $(a, b) \triangle((a / 2, b / 2) \cup(1 / 2+a / 2,1 / 2+b / 2))$ is an interval only if (i) $a=b / 2$ and $b=1 / 2+a / 2$, or (ii) $a=b / 2$ and $b=1 / 2+b / 2$. Thus we have $a=1 / 3$, $b=2 / 3$ or $a=1 / 2, b=1$, which gives $E=(1 / 6,5 / 6), F=(1 / 3,2 / 3)$ or $E=(1 / 4,3 / 4), F=(1 / 2,1)$, respectively.

Corollary 3.8. Let $E$ be of the form $E=[0, a) \cup[b, 1), 0<a<b<1$. Assume that $T_{\phi}$ is not ergodic, where $\phi=1_{E}$. Then $T_{\psi}$ is ergodic, where $\psi=1_{B}, B=(a, b)$. Hence $(a, b) \neq(1 / 6,5 / 6)$ and $(a, b) \neq(1 / 4,3 / 4)$.

Proof of Main Theorem. First, Proposition 3.7 implies that for any interval $E$ such that $E \neq(1 / 6,5 / 6)$ and $E \neq(1 / 4,3 / 4)$ almost every $x$ is a normal number mod 2 with respect to $E$. 
Next, consider $E=[1 / 4,3 / 4]$. Put $F=[1 / 2,1)$. Then $E=F \triangle T^{-1} F$, and $\phi(x)=1_{E}(x)=q(x)+q(T x)(\bmod 2)$, where $q=1_{F}$. Then $\int_{0}^{1} e^{\pi i q} d x=0$, and Fact 2.2(ii) implies $\lim _{N \rightarrow \infty} N^{-1} \sum_{n=1}^{N} d_{n}=1 / 2$ for a.e. $x$. In this case we have mod 2 normality with respect to $E$ even though $1_{E}$ is a coboundary.

There remains the only exceptional case $E=[1 / 6,5 / 6]$ for which the normality $\bmod 2$ with respect to $E$ does not hold. Put $F=[1 / 3,2 / 3]$. Then $E=F \triangle T^{-1} F$. Now use $\int_{0}^{1} e^{\pi i q} d x=1 / 3$.

Remark 3.9. Fig. 3 illustrates the fact that $E=[1 / 6,5 / 6]$ gives a coboundary.

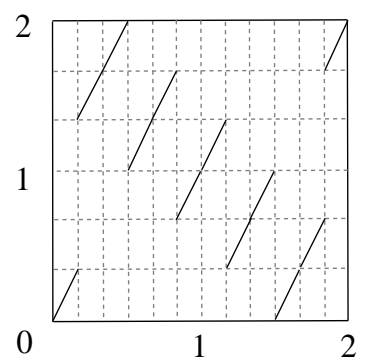

Fig. 3. A nonergodic skew product $T_{\phi}$ generated by the interval $E=[1 / 6,5 / 6] \subset X=$ $[0,1)$ is regarded as an interval map $\widetilde{T}_{\phi}$ on $\widetilde{X}=[0,2)$. It has two ergodic components: $K_{1}=[0,1 / 3] \cup[2 / 3,1] \cup[4 / 3,5 / 3]$ and $K_{2}=\widetilde{X}-K_{1}$.

Acknowledgements. The authors wish to thank the referee for many helpful suggestions.

\section{References}

[1] R. Adler and L. Flatto, Geodesic flows, interval maps, and symbolic dynamics, Bull. Amer. Math. Soc. 25 (1991), 229-334.

[2] Y. Ahn, On compact group extension of Bernoulli shifts, Bull. Austral. Math. Soc. 61 (2000), 277-288.

[3] Y. Ahn and G. H. Choe, On normal numbers mod 2, Colloq. Math. 76 (1998), 161-170.

[4] - - - Spectral types of skewed Bernoulli shift, Proc. Amer. Math. Soc. 128 (2000), 503-510.

[5] G. H. Choe, Spectral types of uniform distribution, ibid. 120 (1994), 715-722.

[6] H. Furstenberg, Strict ergodicity and transformation of the torus, Amer. J. Math. 83 (1961), 573-601.

[7] T. Hamachi, Canonical subrelations of ergodic equivalence relations-subrelations, J. Operator Theory 43 (2000), 3-34.

[8] A. Iwanik, M. Lemańczyk and C. Mauduit, Piecewise absolutely continuous cocycles over irrational rotations, J. London Math. Soc. 59 (1999), 171-187.

[9] A. Iwanik, M. Lemańczyk and D. Rudolph, Absolutely continuous cocycles over irrational rotations, Israel J. Math. 83 (1993), 73-95. 
[10] A. del Junco, M. Lemańczyk and M. K. Mentzen, Semisimplicity, joinings and group extensions, Studia Math. 112 (1995), 141-164.

[11] M. Lemańczyk, M. Mentzen and H. Nakada, Semisimple extensions of irrational rotations, Studia Math. 156 (2003), 31-57.

[12] T. Li and J. A. Yorke, Ergodic transformations from an interval into itself, Trans. Amer. Math. Soc. 235 (1978), 183-192.

[13] K. D. Merrill, Cohomology of step functions under irrational rotations, Israel J. Math. 52 (1985), 320-340.

[14] W. A. Veech, Strict ergodicity in zero dimensional dynamical systems and the Kronecker-Weyl theorem mod 2, Trans. Amer. Math. Soc. 140 (1969), 1-33.

[15] —, Finite group extensions of irrational rotations, Israel J. Math. 21 (1969), 240259.

[16] R. J. Zimmer, Extensions of ergodic group actions, Illinois J. Math. 20 (1976), 373-409.

Department of Mathematics

Korea Advanced Institute

of Science and Technology

Daejeon, South Korea

E-mail: choe@euclid.kaist.ac.kr

Department of Mathematics

Keio University

Yokohama, Japan

E-mail: nakada@math.keio.ac.jp
Department of Mathematics Kyushu University Fukuoka, Japan E-mail: hamachi@math.kyushu-u.ac.jp 